Phys Med Rehabil Clin N Am. 2016 February ; 27(1): 339-355. doi:10.1016/j.pmr.2015.09.002.

\title{
Gait Retraining: Altering the Fingerprint of Gait
}

\author{
Irene S. Davis, PhD, PT, FAPTA, FACSM, FASB ${ }^{1}$ and Erin Futrell, PT, OCS ${ }^{2}$ \\ ${ }^{1}$ Spaulding National Running Center, Department of Physical Medicine and Rehabilitation, \\ Harvard Medical School, Cambridge, MA \\ ${ }^{2} \mathrm{MGH}$ Institute of Health Professions, Center for Interprofessional Studies and Innovation, \\ Charlestown, MA
}

\author{
Keywords \\ running injuries; gait retraining; biofeedback; faded feedback
}

\section{Introduction}

Why would one want to alter someone's natural running gait pattern? In fact, aren't running gait patterns self-optimized for each individual? Unfortunately, this is not the case. Despite Nigg's theory of natural movement patterns, individuals often run with patterns that have been shown to be related to injury. ${ }^{1-4}$ Some patterns have also been associated with reductions in economy, such as increased vertical oscillation, increased stride length and excessive arm motion. ${ }^{5}$ While economy is important to overall running performance, this paper will focus on the retraining of gait patterns with the goal of reducing the risk of running injuries. As running injuries result from musculoskeletal overload, the issues contributing to this overload will first be reviewed, with a focus on biomechanical factors. The inability of strengthening to alter running mechanics will next be discussed. The important components of a retraining program will be described. This will be followed by a review of the literature on gait retraining to reduce injury risk in runners. The paper will conclude with a look into the future of conducting gait retraining in a runner's natural environment using wearable technology.

\section{Factors associated with Running Injuries}

Running is a repetitive activity that typically occurs in a linear, forward direction resulting in a relatively invariant load with each footstrike. As each foot strikes the ground approximately 1000 steps per mile, musculoskeletal tissues of the lower extremity become

\footnotetext{
Correspondence: Irene S. Davis, Spaulding-Cambridge Outpatient Center, 1575 Cambridge St, Cambridge, MA 02138, Phone: 617-952-6835.

Disclosures:

None

Publisher's Disclaimer: This is a PDF file of an unedited manuscript that has been accepted for publication. As a service to our customers we are providing this early version of the manuscript. The manuscript will undergo copyediting, typesetting, and review of the resulting proof before it is published in its final citable form. Please note that during the production process errors may be discovered which could affect the content, and all legal disclaimers that apply to the journal pertain.
} 
susceptible to cumulative overload, leading to overuse injuries. It can be assumed that every runner has a threshold for injury and this threshold is dependent upon a number of factors (Figure 1). One's overall structure and static alignment plays an important role. For instance, foot structure has been implicated in different types of running injuries. ${ }^{6}$ As an example, high arched runners have been shown to be prone to bony injuries, while low arched runners are prone to soft tissue injuries. ${ }^{6}$ While these structural factors need to be considered, they are basically non-modifiable. Dosage is another factor that can influence cumulative load. Training aspects such as how quickly one increases mileage, cross-training, and variance of running surfaces all contribute to the overall dosage of running. Dosage is clearly a modifiable risk factor, but there is a subset of runners, who despite reducing their mileage and resting appropriately are unable to resolve their injury. These are the runners that most likely have an underlying mechanical factor that is not being addressed. This may explain in large part why the most common risk factor for a running injury is a previous injury. ${ }^{7}$ Mechanical factors can be divided into forces (kinetics) and movement patterns (kinematics). A runner exhibiting abnormalities in either of these areas can experience excessive loading in their musculoskeletal system. Runners experiencing both excessive forces and abnormal movement patterns are likely to have an even greater risk for injury.

The impact transient of the vertical ground reaction force is the immediate rise in force shortly after the foot contacts the ground. (Figure 2). A sudden, large impact transient has been associated with injuries. In particular, greater vertical impact peak along with higher average and vertical instantaneous load rates (VALR, VILR) have been linked to tibial stress fractures, plantar fasciitis and patellofemoral pain. ${ }^{2-4}$ In fact, a recent prospective study suggests that having greater VALR increases risk for developing a future running-related injury. ${ }^{8}$ Therefore, increased impacts might be considered one global indicator for injury. This is not surprising, as the musculoskeletal system is comprised of viscoelastic structures that are sensitive to rates of loading. It has long been established in animal studies of bone and cartilage, that repetitive impulsive loads, even those within physiological limits, can have damaging effects on musculoskeletal structures. ${ }^{9-12}$ As stated by these authors, joint wear is determined not simply by the total force applied, but rather by the degree and nature of the loading. ${ }^{10}$ In terms of movement patterns, there are some very common malalignments that clinicians observe in their injured runners. At the foot, these include increased stride length, excessive ankle dorsiflexion or inversion at footstrike, excessive foot eversion, and toe-in and toe-out gait (Figure 3). More proximally, increased genu varum and valgum, and anterior pelvic tilt and contralateral pelvic drop are often seen. However, one of the most common abnormal movement patterns is the combination of excessive hip adduction, internal rotation and pelvic drop (Figure 4). This particular malalignment has been associated with patellofemoral pain, iliotibial band syndrome, and tibial stress fractures in runners. ${ }^{2,4,13}$ Therefore, this malalignment pattern may be considered another global indicator for injury.

\section{Strengthening Alone Is Not Enough}

The most common therapeutic approach to altering faulty movement patterns is to strengthen the muscles that control that movement. For example, excessive hip adduction and internal rotation are often treated by strengthening gluteus medius (a hip abductor) and 
gluteus maximus (a hip external rotator). However, there is little support that strengthening these muscles, without neuromuscular retraining, translates into a change in movement patterns. Snyder et al. examined the effects of strengthening the hip abductors and external rotators on hip and knee mechanics during running in healthy active females. ${ }^{14}$ With the exception of a small increase in hip adduction excursion, the authors noted no changes in hip and knee kinematics following a strengthening program compared to baseline. However, their study did not focus on individuals with abnormal mechanics, which may have limited its ability to examine potential changes in faulty mechanics. However, Mascal et al. reported on a case study involving a female runner with anterior knee pain associated with excessive hip adduction, internal rotation, and contralateral pelvic drop during a step-down maneuver. ${ }^{15}$ These abnormal mechanics were significantly reduced following a 14-week strengthening program. It must be noted that the strengthening program focused on the proper mechanics during the single leg stance and step-down exercises. Therefore, this additional neuromuscular re-education could have influenced the participants' movement patterns and alignment during this specific dynamic task. Willy and Davis examined the effect of a hip-strengthening program on otherwise healthy runners who demonstrated excessive hip adduction with running. ${ }^{16}$ These individuals underwent a very typical 6-week strengthening program addressing the hip abductors and external rotators. These runners demonstrated an approximate $40 \%$ increase in strength of these muscles compared to a control group who did no strengthening. Despite this increase in strength, these runners demonstrated nearly identical post-strengthening hip mechanics compared to their baseline values (Figure 5). Instruction and practice in proper single leg stance activities was also included in the strengthening program. Like Mascal et al., Willy and Davis noted significant improvements in the hip mechanics of the single leg squat. However, these changes did not translate to alterations during the higher demand activity of running. This finding clearly underscores the need for the retraining to be activity-specific.

\section{Brief History of Gait Retraining}

The idea of altering gait patterns using feedback is not novel. The earliest forms of feedback were limb load monitors placed within the shoe of a patient. ${ }^{17-19}$ The aim of this type of feedback was to produce an equal load distribution between lower extremities during gait. Electromyography is one of the most widely used forms of feedback reported in the literature. Reports of improvements in gait symmetry in terms of spatio-temporal parameters and joint motion patterns have been reported with use of EMG feedback. ${ }^{20-24}$ Feedback on joint angles has been provided through the use of electrogoniometers for patients with genu recurvatum. ${ }^{25-27}$ An overwhelming majority of these studies have reported successful results. However, none of these assessed the persistence or retention of these gait patterns over time, and the majority of these studies involved patients with neurologic disorders.

Reports of real-time feedback training then began to emerge in the orthopedic literature. In 2005, White and Lifeso provided real time force feedback from an instrumented treadmill to patients who walked asymmetrically following a hip replacement. ${ }^{28}$ They reported a significant improvement in symmetry of ground reaction forces at weight acceptance following an 8-week (3 times per week) gait retraining program. In a related study, Dingwell used an instrumented treadmill to improve the gait patterns of a group of unilateral, trans- 
tibial amputees. ${ }^{29}$ Prior to the training, asymmetries in the measured parameters were 4.6 times greater in the amputee group compared to the control group. These asymmetries were significantly reduced following the training. However, the long-term persistence of these changes was not monitored in either study and both investigations focused on walking gait. The physical demands of running are greater than walking, thus increasing the challenges of altering habitual running gait patterns.

\section{Components of a Retraining Program}

Altering any motor pattern that has become habituated over many years can be difficult. This is especially true when you consider retraining a runner. Runners strike the ground approximately 1000 steps/mile. An individual who runs 20 miles per week can log over 1 million footstrikes per year, or 10 million footstrikes if they have been running for 10 years. Altering a motor pattern that has been reinforced over millions of cycles takes both guidance and practice to alter. In a review on motor control principles, Winstein defined motor learning as a set of internal processes associated with practice or experience leading to a relatively permanent change in the capability for responding. These processes are thought to be complex central nervous system phenomena whereby sensory and motor information is organized and integrated. ${ }^{30}$ This author suggests that learning a new motor program is enhanced with feedback provided in two phases. During the first, acquisition phase, extrinsic feedback is provided on a prescribed schedule. This helps to develop the connection between the extrinsic feedback (e.g., real time video of individual running) with the internal sensory cues (i.e., proprioception) associated with the desired motor pattern. During the second, transfer phase, the feedback is removed in a systematic fashion. The fading of this feedback prevents the reliance on it, and enhances the internalization, and thus learning of the new motor pattern.

While altering a motor pattern may be desired, it is also likely to alter the loads on the musculoskeletal system. For example, retraining a runner to become a forefoot striker in order to reduce vertical impacts, reduces the load to the knee, but increases the load to the calf. It is critical to fortify the calf musculature in order to reduce the risk of an overuse injury to this area. Therefore, it is important to include a specific strengthening program in any gait retraining intervention to anticipate increased demands to other components of the kinetic chain.

\section{Altering movement patterns in runners}

One of the earliest gait retraining studies in runners was conducted by Messier and Cirillo in 1989. ${ }^{31}$ In this study, runners were seen 3 times per week for 5 weeks. Prior to each training session, subjects were shown a videotape of their running and were instructed on the features of gait they were to modify. These were subject-specific mechanics and included characteristics such as excessive vertical oscillation, over-striding, excessive trunk lean and excessive arm motion. This group of runners significantly altered the desired kinematic gait variables compared to a control group who received no feedback prior to their training sessions. This study demonstrates that runners are indeed able to alter their mechanics with 
retraining. However, persistence of these changes following training was not examined, and it is therefore unclear whether true motor learning occurred.

With the development of real time motion analysis systems, gait retraining could be augmented with real-time feedback on specific joint angles. Armed with this technology, Noehren et al. investigated 10 female runners with a history of patellofemoral pain (average duration of 75.7 months) who demonstrated excessive hip adduction. ${ }^{32}$ These runners underwent 8 sessions (over 2 weeks) of gait retraining, learning how to activate the gluteal muscles in order to reduce hip adduction. Markers were placed on the lower extremities so that hip adduction could be monitored in real time. The hip adduction angle of the most involved side was displayed on a monitor in front of the runner during the stance phase of each footstrike. This angle was superimposed onto a graph of a normal hip adduction trajectory with a shaded region denoting $+/-1$ standard deviation of this mean value (Figure 6). Runners were asked to keep their hip adduction angle within the shaded region by modulating the activation of their gluteal muscles. The authors were highly interested in the persistence of these changes. Therefore, they utilized a paradigm that incorporated the concepts of motor learning that were described by Winstein. ${ }^{30}$ Subjects gradually increased their run time from 10 to 30 minutes over the 8 sessions. During the first 4 sessions, runners were provided feedback 100\% of the time (acquisition phase). During the last 4 sessions, feedback was faded (transfer phase) such that they received only 3 minutes of feedback during the last session ( 1 in the beginning, 1 in the middle and 1 in the end) (Figure 7). Their gait data were examined at baseline, immediately post-training, and at 1- month follow-up. As can be seen in Figure 8, runners were able to reduce their hip adduction, internal rotation and contralateral pelvic drop following gait retraining. Additionally, they were able to maintain this at the 1-month follow-up. Controlling their hips better during running also led to reduced vertical load rates, which have been shown to be related to patellofemoral pain. ${ }^{33}$ Along with persistency, an indication of learning is the ability to transfer the new motor pattern to an untrained activity. ${ }^{34-36}$ These authors noted that hip alignment also improved during the activity of a single leg squat. Most important to the patients, was a complete resolution of pain and significant improvement in overall function.

While these real time motion analysis systems can provide powerful feedback, it is unlikely they will be readily available in clinical settings. Therefore, Willy et al. repeated the study of Noehren et al. using a similar female patient population with chronic patellofemoral pain (average pain duration 51 months). ${ }^{32,37}$ However, they simply used a mirror as the real time feedback. They used an identical faded feedback design, and their subjects' post-training improvements in hip mechanics were very similar to Noehren et al. ${ }^{32}$ Additionally, investigators found these changes to persist at both 1 and 3-month follow-ups. As with Noehren et al. the reductions in hip adduction transferred to other functional activities including a single leg squat and a step down maneuver. ${ }^{32}$ Again, pain was resolved and function restored and all runners returned to at least their pre-training mileage by the 3month follow-up. Six of the 10 subjects also reported running higher mileage, and attributed this to a decrease in their knee pain following gait retraining. 


\section{Altering loading patterns and footstrike patterns in runners}

Gait retraining has also been used to reduce high impact loading in runners. Crowell and Davis recruited 10 healthy runners with high tibial shock (acceleration) to engage in a retraining study to reduce impact loading. ${ }^{38}$ These runners had a lightweight accelerometer affixed tightly to their distal medial tibia above the medial malleolus (Figure 9). They ran on a treadmill with a monitor in front of them displaying the tibial accelerations with each footstrike. They were instructed to land softly and keep their tibial shock values below a line placed on the screen that denoted a normal level (Figure 9). They were simply told to land as softly as possible without any specific instruction on how to do so. These authors used a similar feedback paradigm as Noehren et al. and Willy et al. ${ }^{32,37}$ All runners reported that the new gait pattern felt natural by the sixth retraining session. Additionally, runners were able to reduce their tibial shock, by $48 \%$ on average. Vertical instantaneous and average load rates were also reduced by $34 \%$ and $32 \%$. These changes persisted at the 1-month follow-up.

Running with a forefoot strike pattern has been shown to reduce impact loading and can be an effective approach to reducing patellofemoral joint pain. Patellofemoral pain is related, in part, to increases in the patellofemoral contact stresses. ${ }^{39,40}$ It has been shown that a forefoot strike results in a reduced vertical force at early stance due to lower rates of loading. This is coupled with an increased knee flexion angle (thus greater patellofemoral contact area) in early stance. These combined biomechanical factors lead to a reduced patellofemoral contact stress and potentially less pain. ${ }^{41}$ In a case series of three patients with chronic patellofemoral pain, Cheung and Davis aimed to transition the runners from a rearfoot strike pattern to a forefoot strike pattern. ${ }^{42}$ Instrumented insoles were used to provide an audible sound when the runners landed on their heels. Runners underwent 8 sessions of gait retraining using the faded feedback design of others. ${ }^{32,37}$ They were able to successfully transition these runners to a forefoot strike pattern that persisted at both 1 and 3 months. This resulted in significant reductions in vertical impact peaks, as well as vertical average and instantaneous load rates. Most importantly, these runners demonstrated marked improvements in their pain and function.

In addition to exacerbating patellofemoral pain, landing on one's heel increases the demand of the anterior muscles of the lower leg and can contribute to compartment syndrome. When the demand becomes excessive, such as when landing with increased ankle dorsiflexion, the muscles hypertrophy faster than the fascial compartments can accommodate. This causes increased pressure to develop in the compartment, which can damage the nerves and vessels in this area. A common approach to this problem is to surgically release the fascia to relieve the pressure in the compartment. However, this can lead to scar tissue development, and result in a recurrence of the compartment syndrome. Diebel et al. recruited a group of 10 West Point cadets who were diagnosed with compartment syndrome and were indicated for a surgical release. ${ }^{43}$ Instead, they underwent a 6 -week (3 times per week) program of forefoot run training that included running drills, cadence practice, video recording, metronome feedback, and barefoot running. At the end of 6 weeks, intracompartmental pressures had returned to normal, symptoms had resolved, vertical load rates were reduced, 
and cadence was significantly improved. All runners were able to return to their pre-injury running, all increased their run times, and all were able to avoid a surgical release.

The recent shift towards adopting a more anterior foot strike pattern during running has led to transition studies using healthy individuals. Warne et al. conducted a 4-week intervention to gradually transition to a forefoot strike pattern in minimal footwear (Vivo Barefoot "Evo", Terra Plana, London, UK). ${ }^{44}$ The program included technique instruction, a prescribed schedule of running progression, as well as a stretching and strengthening component focused on the foot and ankle. At the end of the 4-week program, 8 of the 10 runners had transitioned successfully to a forefoot strike pattern. However, it is unclear whether motor learning occurred, as there were no follow-up assessments to determine persistence. McCarthy et al. conducted a similar intervention study. ${ }^{45}$ This group of 9 rearfoot strike runners was provided minimal footwear (Vibram FiveFingers Classic). They were instructed in a foot-strengthening program and were then given a prescribed 12-week progressive running program to be conducted independently. A control group of 10 runners continued to run their normal mileage in their standard footwear. At the end of the training, the intervention group demonstrated a significantly greater plantarflexed ankle at footstrike indicating a forefoot strike pattern. However, as with Warne et al., there was no assessment of short or long-term persistence of these changes. ${ }^{44}$ The persistence and retention of these changes, especially in the long-term, must be evaluated in order to establish gait retraining as an effective method to alter foot strike pattern and provide a treatment strategy to reduce running injuries.

\section{Case study of transitioning intervention with long-term follow up}

A 24 year-old female runner had running-related deep knee joint pain and lateral knee pain on the right for approximately 7 months prior to being seen as a patient at the Spaulding National Running Center (SNRC) in Cambridge, MA. At the time of her initial evaluation, extended walking, stairs, and squatting were all painful. She had also tried swimming and cycling, and both were painful in the knee. Prior to her time at the SNRC, she underwent a course of physical therapy that consisted primarily of stretching and strengthening, which did not improve her symptoms. She was referred back to her physician due to lack of progress. She had radiographs and an MRI of her knee, which were negative for pathology. She stated that her knee was painful at all times during activities of daily living and reported a verbal analog pain scale of $2 / 10$ at best and $7 / 10$ at worst.

This patient ran with a rearfoot strike (RFS) bilaterally, exhibiting vertical impact peaks (Figure 10A). She demonstrated weakness of her foot and ankle muscles bilaterally, only completing 5 heel raises on the left and 2 on the right. When asked to run with a forefoot strike (FFS) pattern while barefoot, her right deep knee joint pain immediately reduced from $7 / 10$ to $5 / 10$ and her right lateral knee pain reduced from $5 / 10$ to $0 / 10$. Vertical impact peaks were immediately eliminated (Figure 10B) and vertical instantaneous and average load rates were decreased by $36 \%$ and $40 \%$ on the injured right side, respectively (Figure 11). These reductions are consistent with results we published in a group of 49 patients seen in the SNRC clinic. ${ }^{46}$ 
This runner underwent a 7-week program consisting of 4 weeks of foot and lower leg progressive strengthening and 8 sessions of gait retraining using a faded feedback design to transition to a FFS pattern. At the end of the sessions, this patient was running for 30 minutes with a consistent FFS pattern and no pain. She was instructed to increase her mileage by $10 \%$ per week following discharge.

The patient returned for a 1-year follow-up. Since her discharge, she has continued to run in minimal shoes an average of 15 miles per week and she has been pain-free. She now runs with a FFS with $10 \%$ reduced stride length compared to her baseline at time of injury. Her vertical instantaneous and average load rates in the minimal footwear were reduced by $36 \%$ and $52 \%$ respectively on the injured right side (Figure 11). Not noted in the graph is the reduction of her vertical instantaneous and average load rates of $58 \%$ and $63 \%$ on the left, uninjured side, indicating the transfer of mechanics to the contralateral leg. This case study supports the association of impacts with knee pain, as well as the reduction of knee pain with reduction of the impacts. ${ }^{33,41,42}$ It also supports the hypotheses that transitioning to a FFS pattern with minimal footwear resulted in reduction of vertical impact loading in both the short and long term follow-up. This case illustrates that the transition to a FFS pattern can be accomplished without injury when the runner is adequately prepared. Additionally, it signifies that these changes can be maintained in the long term. Finally, it demonstrates that this approach was successful in eliminating injury in a runner who had undergone a previous unsuccessful course of physical therapy.

\section{Monitoring runners in their natural environment}

The majority of studies of running mechanics and gait retraining have been conducted in the laboratory, which is clearly an artificial setting. Data are typically collected overground, as a runner traverses a runway with a force plate at its center. Depending on the length of the runway, the ability to reach a typical running speed and maintain it for a number of steps can be limited. Additionally, the runner's foot must land fully on the force plate, increasing the chance for targeting. Alternatively, data can be collected on a treadmill. While kinematics between overground and treadmill running have been deemed similar, the treadmill constrains the runner to a single speed, unlike running outdoors where speed is likely to vary. ${ }^{47}$ Both overground and treadmill running do not mimic the natural environment of banked surfaces, hills, and other variations in terrain. Finally, the mechanics of a runner who knows they are being observed may not be representative of their true mechanics. Therefore, there is great ecologic value in studying runners in their natural environments.

Willy et al. has demonstrated a fairly simple way to train a runner to increase their cadence outside of the laboratory. ${ }^{48}$ These authors utilized a wireless accelerometer that transmitted data to a computerized watch that displayed running time, distance, and strides/minute during a running session. Sixteen runners, screened for high impact loading were included in the intervention while 14 runners served as the controls. Following a baseline instrumented gait analysis in the laboratory, runners in the intervention group were instructed to complete 8 typical runs and to aim for increasing their cadence by $7.5 \% .{ }^{49}$ Subjects were instructed to use the feedback when they felt they needed it, as self-controlled feedback has recently been shown to improve motivation. ${ }^{30}$ An intermittent feedback design was incorporated whereby 
the self-controlled feedback was provided on alternate days. ${ }^{30}$ Data were collected in the laboratory at baseline, following the retraining, and at a 1-month follow-up. These authors found that runners were able to increase their cadence by approximately $8 \%$. This resulted in an approximate $18 \%$ reduction in vertical load rates. While this is a significant decrease, the post-training values of $83 \mathrm{BW} / \mathrm{s}$ are still considered high for the speed they were running. ${ }^{2}$ Other measures to reduce impact, such as transitioning to a forefoot strike pattern, may be preferred when aiming to reduce load rates as much as possible in an injured population. Additionally, peak hip adduction, a common malalignment in runners, was reduced by 2.5 degrees, or $14 \%$ from pre-intervention values. This was consistent with the findings of Heidersheidt et al., who reported similar reductions when running with a higher cadence. ${ }^{49}$ The changes these authors noted persisted at the 1-month follow-up. This study also assessed changes in cumulative load with alterations in gait patterns. Despite the increase in number of steps, the cumulative eccentric knee work over the course of a run was lower with the increased cadence. It should be noted that all gait assessments were conducted in the laboratory under the observation of the investigators. As such, this gait may not be representative of the runner's gait in their natural environment.

The development of mobile monitoring devices has made it possible to analyze aspects of running mechanics outside of the laboratory environment. Inertial measurement units (IMUs), like the one depicted in Figure 12a, allow the study of accelerometry, as well as joint kinematics. Additionally, instrumented insoles are emerging in the market to detect foot pressures during running (Figure 12b). These devices have Bluetooth ${ }^{\circledR}$ capability that can wirelessly monitor, record, and provide feedback on a variety of biomechanical measures in real time and in multiple environments. These developments will help advance gait retraining research that is more ecologically valid.

\section{Summary}

In summary, we have an incredible untapped abiity to adapt out own movement patterns. The concept of retraining motor patterns is not new. However, there continues to be some skepticism of the ability to alter the relatively automatic function of both walking and running gait. In terms of running, there is evidence that links mechanics with injury. This provides the justification for altering these mechanics. Increased hip adduction and vertical impact loading have been most commonly associated with injury. As such, most retraining studies are focused around these issues. However, gait retraining principles can be applied to any gait abnormality that is believed to be related to an overuse musculoskeletal problem. While all of the retraining studies to date have resulted in improvements in gait mechanics, the optimal retraining paradigms are likely yet to be determined. Studies need to compare the efficacy of different types of feedback (visual, haptic, auditory) as well as different feedback schedules. The majority of studies involved small subject numbers. It is important to know whether the success of these small studies translates into larger study populations. Additionally, long-term follow-ups (i.e. 1 year and beyond) are needed to establish the retention and persistence of gait changes. Studies need to include more tests that examine the transfer of new patterns to other untrained activities. Finally, we need to monitor runners and retrain their gait patterns in their natural environments. In conclusion, much more work is needed in order to understand the optimal way to retrain gait patterns in runners. The 
human body has an incredible ability to adapt. To provide individuals with the ability to alter faulty movement patterns in ways that can reduce injury risk is a very powerful tool.

\section{References}

1. Nigg BM, Baltich J, Hoerzer S, Enders H. Running shoes and running injuries: mythbusting and a proposal for two new paradigms: "preferred movement path" and "comfort filter". Br J Sports Med. Jul.2015 bjsports - 2015-095054. 10.1136/bjsports-2015-095054

2. Milner CE, Ferber R, Pollard CD, Hamill J, Davis IS. Biomechanical factors associated with tibial stress fracture in female runners. Med Sci Sports Exerc. 2006; 38(2):323-328.10.1249/01.mss. 0000183477.75808.92 [PubMed: 16531902]

3. Pohl MB, Hamill J, Davis IS. Biomechanical and anatomic factors associated with a history of plantar fasciitis in female runners. Clin J Sport Med Off J Can Acad Sport Med. 2009; 19(5):372376.10.1097/JSM.0b013e3181b8c270

4. Noehren B, Hamill J, Davis I. Prospective evidence for a hip etiology in patellofemoral pain. Med Sci Sports Exerc. 2013; 45(6):1120-1124.10.1249/MSS.0b013e31828249d2 [PubMed: 23274607]

5. Williams KR, Cavanagh PR. Relationship between distance running mechanics, running economy, and performance. J Appl Physiol. 1987; 63(3):1236-1245. [PubMed: 3654469]

6. Williams DS 3rd, McClay IS, Hamill J. Arch structure and injury patterns in runners. Clin Biomech Bristol Avon. 2001; 16(4):341-347.

7. Saragiotto BT, Yamato TP, Hespanhol Junior LC, Rainbow MJ, Davis IS, Lopes AD. What are the Main Risk Factors for Running-Related Injuries? Sports Med. 2014; 44(8):1153-1163.10.1007/ s40279-014-0194-6 [PubMed: 24809248]

8. Zadpoor AA, Nikooyan AA. The relationship between lower-extremity stress fractures and the ground reaction force: A systematic review. Clin Biomech. 2011; 26(1):23-28.10.1016/ j.clinbiomech.2010.08.005

9. Radin EL, Paul IL. Response of Joints to Impact Loading. I. In Vitro Wear. Arthritis Rheum. 1971; 14(3):356-362.10.1002/art.1780140306 [PubMed: 5562019]

10. Radin EL, Parker HG, Pugh JW, Steinberg RS, Paul IL, Rose RM. Response of Joints to Impact Loading - III - Relationship Between Trabecular Microfractures and Cartilage Degeneration. J Biomech. 1973; 6:51-57. [PubMed: 4693868]

11. Burr DB, Milgrom C, Boyd RD, Higgins WL, Robin G, Radin EL. Experimental stress fractures of the tibia. Biological and mechanical aetiology in rabbits. J Bone Joint Surg Br. 1990; 72(3):370 375. [PubMed: 2341429]

12. Schaffler MB, Radin EL, Burr DB. Mechanical and Morphological Effects of Strain Rate on Fatigue of Compact Bone - Schaffler, Radin, Burr - 1989.pdf. Bone. 1989; 10(3):207-214. [PubMed: 2803855]

13. Ferber R, Noehren B, Hamill J, Davis IS. Competitive female runners with a history of iliotibial band syndrome demonstrate atypical hip and knee kinematics. J Orthop Sports Phys Ther. 2010; 40(2):52-58.10.2519/jospt.2010.3028 [PubMed: 20118523]

14. Snyder KR, Earl JE, O'Connor KM, Ebersole KT. Resistance training is accompanied by increases in hip strength and changes in lower extremity biomechanics during running. Clin Biomech. 2009; 24(1):26-34.10.1016/j.clinbiomech.2008.09.009

15. Mascal CL, Landel R, Powers C. Management of patellofemoral pain targeting hip, pelvis, and trunk muscle function: 2 case reports. J Orthop Sports Phys Ther. 2003; 33(11):647-660. [PubMed: 14669960]

16. Willy RW, Davis IS. The effect of a hip-strengthening program on mechanics during running and during a single-leg squat. J Orthop Sports Phys Ther. 2011; 41(9):625-632.10.2519/jospt. 2011.3470 [PubMed: 21765220]

17. Wannstedt GT, Herman RM. Use of augmented sensory feedback to achieve symmetrical standing. Phys Ther. 1978; 58(5):553-559. [PubMed: 643934] 
18. Seeger BR, Caudrey DJ. Biofeedback therapy to achieve symmetrical gait in children with hemiplegic cerebral palsy: long-term efficacy. Arch Phys Med Rehabil. 1983; 64(4):160-162. [PubMed: 6838342]

19. Seeger BR, Caudrey DJ, Scholes JR. Biofeedback therapy to achieve symmetrical gait in hemiplegic cerebral palsied children. Arch Phys Med Rehabil. 1981; 62(8):364-368. [PubMed: 7259468]

20. Burnside I, Tobias H, DB. Electromyographic feedback in the remobilization of stroke patients: a controlled trial. Arch Phys Med Rehabil. 1982; 63(5):217-222. [PubMed: 7041850]

21. Colborne GR, Olney SJ, Griffin MP. Feedback of ankle joint angle and soleus electromyography in the rehabilitation of hemiplegic gait. Arch Phys Med Rehabil. 1993; 74(10):1100-1106. [PubMed: 8215864]

22. Colborne G, Wright F, Naumann S. Feedback of triceps surae EMG in gait of children with cerebral palsy: a controlled study. Arch Phys Med Rehabil. 1994; 75(1):40-45. [PubMed: 8291961]

23. Petrofsky JS. The Use of Electromyogram Biofeedback to Reduce Trendelenburg Gait. Eur J Appl Physiol. 2001; 85:491-495.10.1007/s004210100466 [PubMed: 11606020]

24. Intiso D, Santilli V, Grasso MG, Rossi R, Caruso I. Rehabilitation of walking with electromyographic biofeedback in foot-drop after stroke. Stroke. 1994; 25(6):11891192.10.1161/01.STR.25.6.1189 [PubMed: 8202978]

25. Hogue R, McCandless S. Genu recurvatum: auditory biofeedback treatment for adult patients with stroke or head injuries. Arch Phys Med Rehabil. 1983; 64(8):368-370. [PubMed: 6882176]

26. Morris M, Matyas T, TMB, PAG. Electrogoniometric feedback: its effect on genu recurvatum in stroke. Arch Phys Med Rehabil. 1992; 73(12):1147-1154. [PubMed: 1463378]

27. Olney SJ, Colborne GR, Martin CS. Joint Angle Feedback and Biomechanical Gait Analysis in Stroke Patients: A Case Report. Phys Ther. 1989; 69(10):863-870. [PubMed: 2780812]

28. White SC, Lifeso RM. Altering Asymmetric Limb Loading After Hip Arthroplasty Using RealTime Dynamic Feedback When Walking. Arch Phys Med Rehabil. 2005; 86(10):19581963.10.1016/j.apmr.2005.04.010 [PubMed: 16213238]

29. Dingwell JB, Davis BL, Frazder DM. Use of an Instrumental Treadmill for Real-Time Gait Symmetry Evaluation and Feedback in Normal and Trans-Tibial Amputee Subjects - Dingwell, Davis, Frazder. Prosthet Orthot Int. 1996; 20(2):101-110.10.3109/03093649609164426 [PubMed: 8876003]

30. Winstein CJ. Knowledge of results and motor learning--implications for physical therapy. Phys Ther. 1991; 71(2):140-149. [PubMed: 1989009]

31. Messier SP, Cirillo KJ. Effects of a Verbal and Visual Feedback System on Running Technique, Perceived Exertion and Running Economy in Female Novice Runners. J Sports Sci. 1989; 7:113126. [PubMed: 2681819]

32. Noehren B, Scholz J, Davis I. The effect of real-time gait retraining on hip kinematics, pain and function in subjects with patellofemoral pain syndrome. Br J Sports Med. 2011; 45(9):691696.10.1136/bjsm.2009.069112 [PubMed: 20584755]

33. Davis IS, Bowser BJ, Hamill J. Vertical Impact Loading in Runners with a History of Patellofemoral Pain Syndrome. Med Sci Sports Exerc. 2010; 42(5):682.

34. Salmoni AW, Schmidt RA, Walter CB. Knowledge of results and motor learning: A review and critical reappraisal. Psychol Bull. 1984; 95(3):355-386.10.1037/0033-2909.95.3.355 [PubMed: 6399752]

35. Schmidt RA, White JL. Evidence for an Error Detection Mechanism In Motor Skills. J Mot Behav. 1972; 4(3):143-153.10.1080/00222895.1972.10734930 [PubMed: 23952592]

36. Sherwood DE, Lee TD. Schema Theory: Critical Review and Implications for the Role of Cognition in a New Theory of Motor Learning. Res Q Exerc Sport. 2003; 74(4):376382.10.1080/02701367.2003.10609107 [PubMed: 14768838]

37. Willy RW, Scholz JP, Davis IS. Mirror gait retraining for the treatment of patellofemoral pain in female runners. Clin Biomech Bristol Avon. Aug.2012 10.1016/j.clinbiomech.2012.07.011

38. Crowell HP, Davis IS. Gait retraining to reduce lower extremity loading in runners. Clin Biomech Bristol Avon. 2011; 26(1):78-83.10.1016/j.clinbiomech.2010.09.003 
39. Farrokhi S, Keyak JH, Powers CM. Individuals with patellofemoral pain exhibit greater patellofemoral joint stress: a finite element analysis study. Osteoarthr Cartil OARS Osteoarthr Res Soc. 2011; 19(3):287-294.10.1016/j.joca.2010.12.001

40. Heino Brechter J, Powers C. Patellofemoral stress during walking in persons with and without patellofemoral pain. Med Sci Sports Exerc. 2002; 34(10):15821593.10.1097/00005768-200210000-00009 [PubMed: 12370559]

41. Bonacci J, Vicenzino B, Spratford W, Collins P. Take your shoes off to reduce patellofemoral joint stress during running. Br J Sports Med. Jul.2013 10.1136/bjsports-2013-092160

42. Cheung RTH, Davis IS. Landing pattern modification to improve patellofemoral pain in runners: a case series. J Orthop Sports Phys Ther. 2011; 41(12):914-919.10.2519/jospt.2011.3771 [PubMed: 22031595]

43. Diebal AR, Gregory R, Alitz C, Gerber JP. Forefoot Running Improves Pain and Disability Associated With Chronic Exertional Compartment Syndrome. Am J Sports Med. 2012; 40(5): 1060-1067.10.1177/0363546512439182 [PubMed: 22427621]

44. Warne JP, Kilduff SM, Gregan BC, Nevill AM, Moran KA, Warrington GD. A 4-week instructed minimalist running transition and gait-retraining changes plantar pressure and force. Scand J Med Sci Sports. 2013 n/a-n/a. 10.1111/sms. 12121

45. McCarthy C, Fleming N, Donne B, Blanksby B. 12 weeks of simulated barefoot running changes foot-strike patterns in female runners. Int J Sports Med. 2014; 35(5):443-450.10.1055/ s-0033-1353215 [PubMed: 24048910]

46. Samaan CD, Rainbow MJ, Davis IS. Reduction in ground reaction force variables with instructed barefoot running. J Sport Health Sci. 2014; 3(2):143-151.10.1016/j.jshs.2014.03.006

47. Fellin RE, Manal K, Davis IS. Comparison of lower extremity kinematic curves during overground and treadmill running. J Appl Biomech. 2010; 26(4):407-414. [PubMed: 21245500]

48. Willy RW, Buchenic L, Rogacki K, Ackerman J, Schmidt A, Willson JD. In-field gait retraining and mobile monitoring to address running biomechanics associated with tibial stress fracture. Scand J Med Sci Sports. Mar.2015 n/a-n/a. 10.1111/sms.12413

49. Heiderscheit BC, Chumanov ES, Michalski MP, Wille CM, Ryan MB. Effects of step rate manipulation on joint mechanics during running. Med Sci Sports Exerc. 2011; 43(2):296302.10.1249/MSS.0b013e3181ebedf4 [PubMed: 20581720] 
- In terms of running, there is evidence that links mechanics with injury.

- This provides the justification for altering these mechanics.

- Increased hip adduction and vertical impact loading have been most commonly associated with injury.

- Much more work is needed in order to understand the optimal way to retrain gait patterns in runners.

- The human body has an incredible ability to adapt.

- To provide individuals with the ability to alter faulty movement patterns in ways that can reduce injury risk is a very powerful tool. 


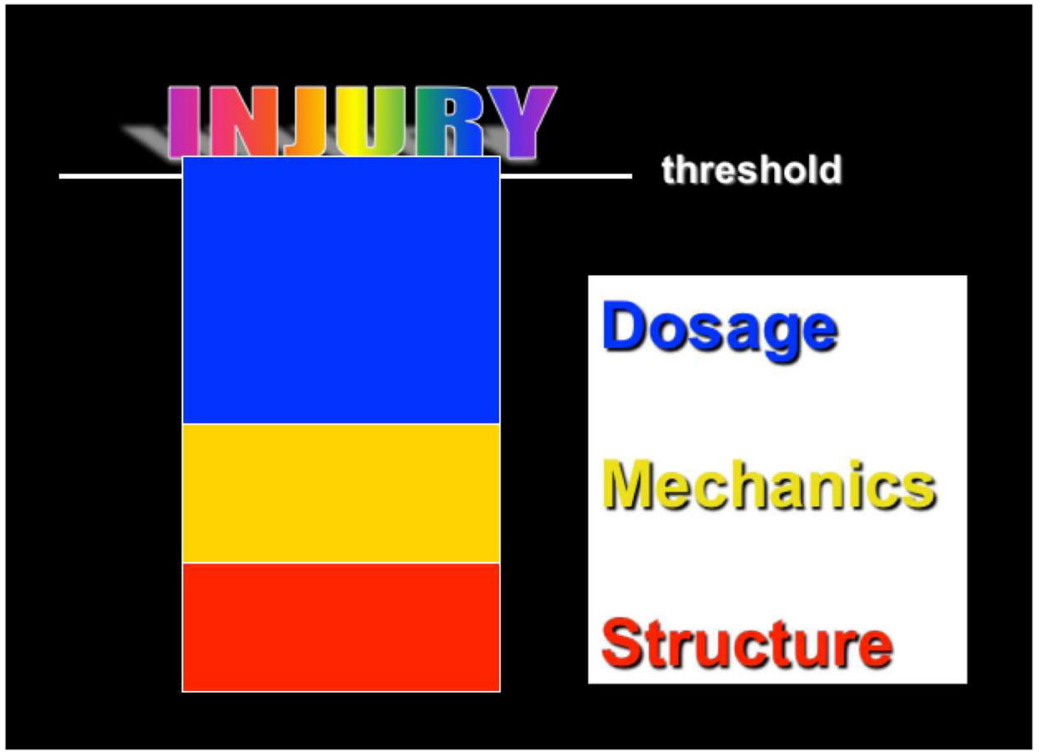

Figure 1.

Basic factors contributing to running injuries. Any one or combination of factors can cause a runner to reach their injury threshold. 


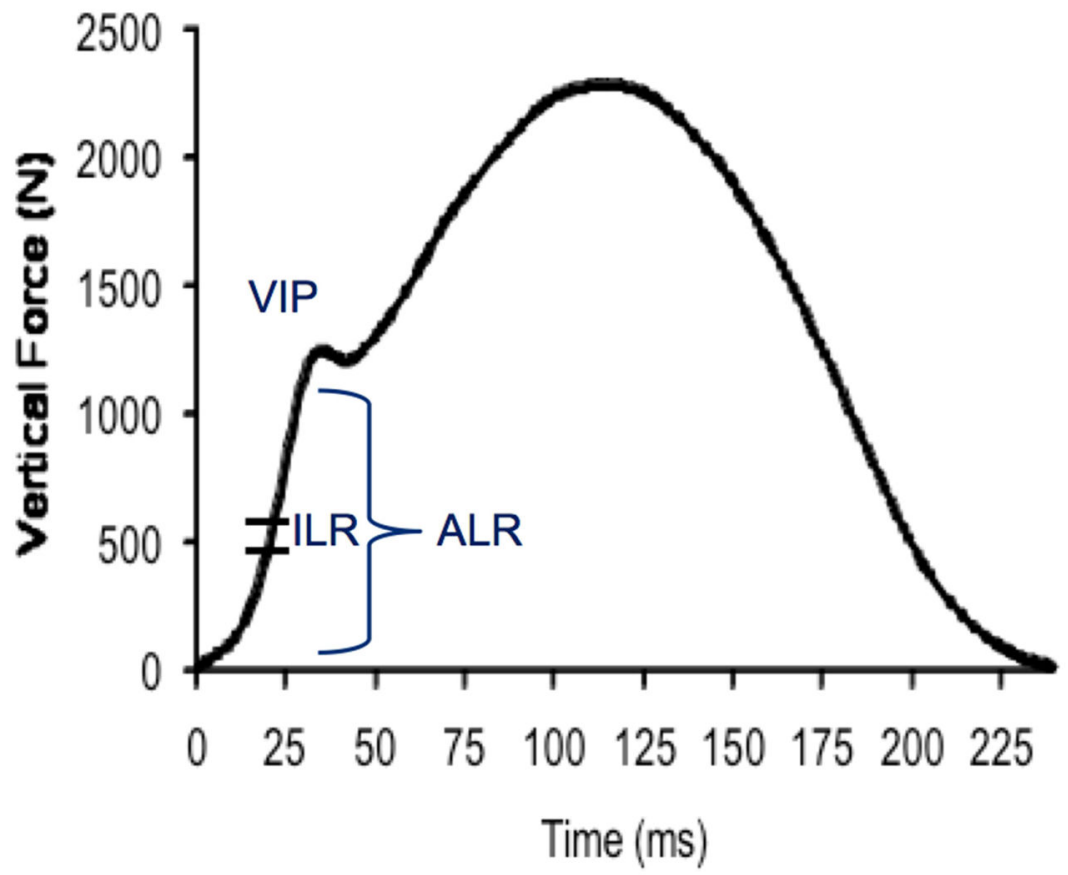

Figure 2.

Calculation of vertical load rates from the vertical ground reaction force during the stance phase of running. The average load rate (ALR) is calculated as the slope of the early impact transient over its most linear portion (between 20-80\% of the vertical impact peak (VIP)). The instantaneous load rate (ILR) is calculated as the peak slope between any two successive points along the same region of interest. 


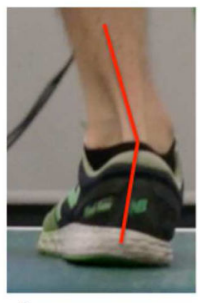

A

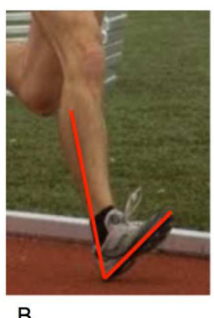

B

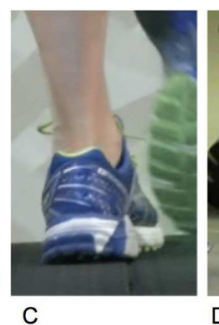

C

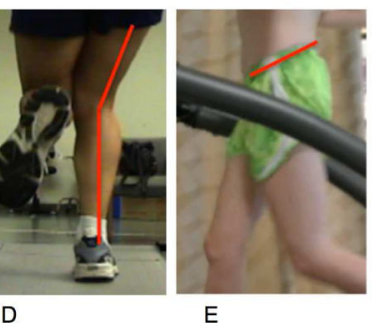

$\mathrm{E}$

Figure 3.

Malalignments seen in runners. A. Excessive peak rearfoot pronation. B. Excessive ankle dorsiflexion at footstrike. C. Toe-in during stance. D. Genu valgum during stance. E. Excessive anterior tilt of the pelvis. 


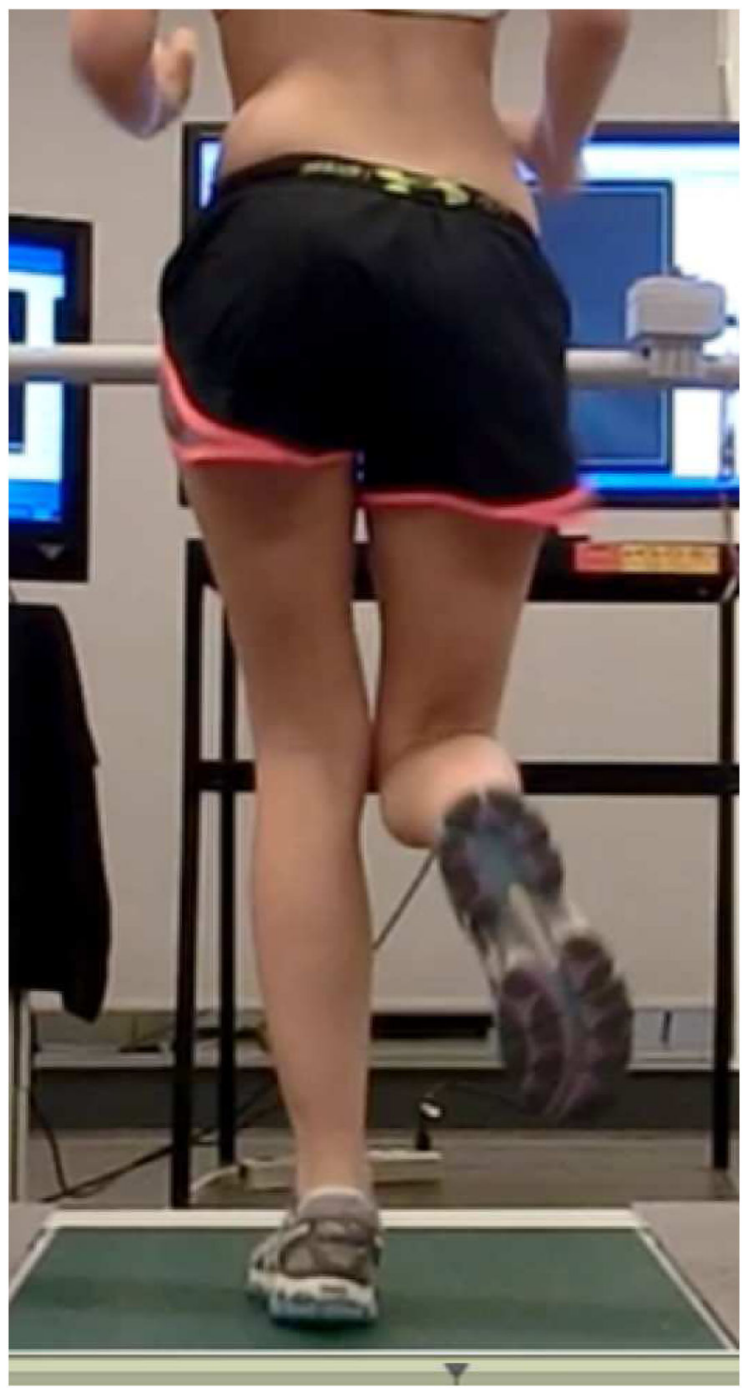

Figure 4.

One of the most common malalignments seen in runners involving excessive contralateral pelvic drop, hip adduction and internal rotation. 

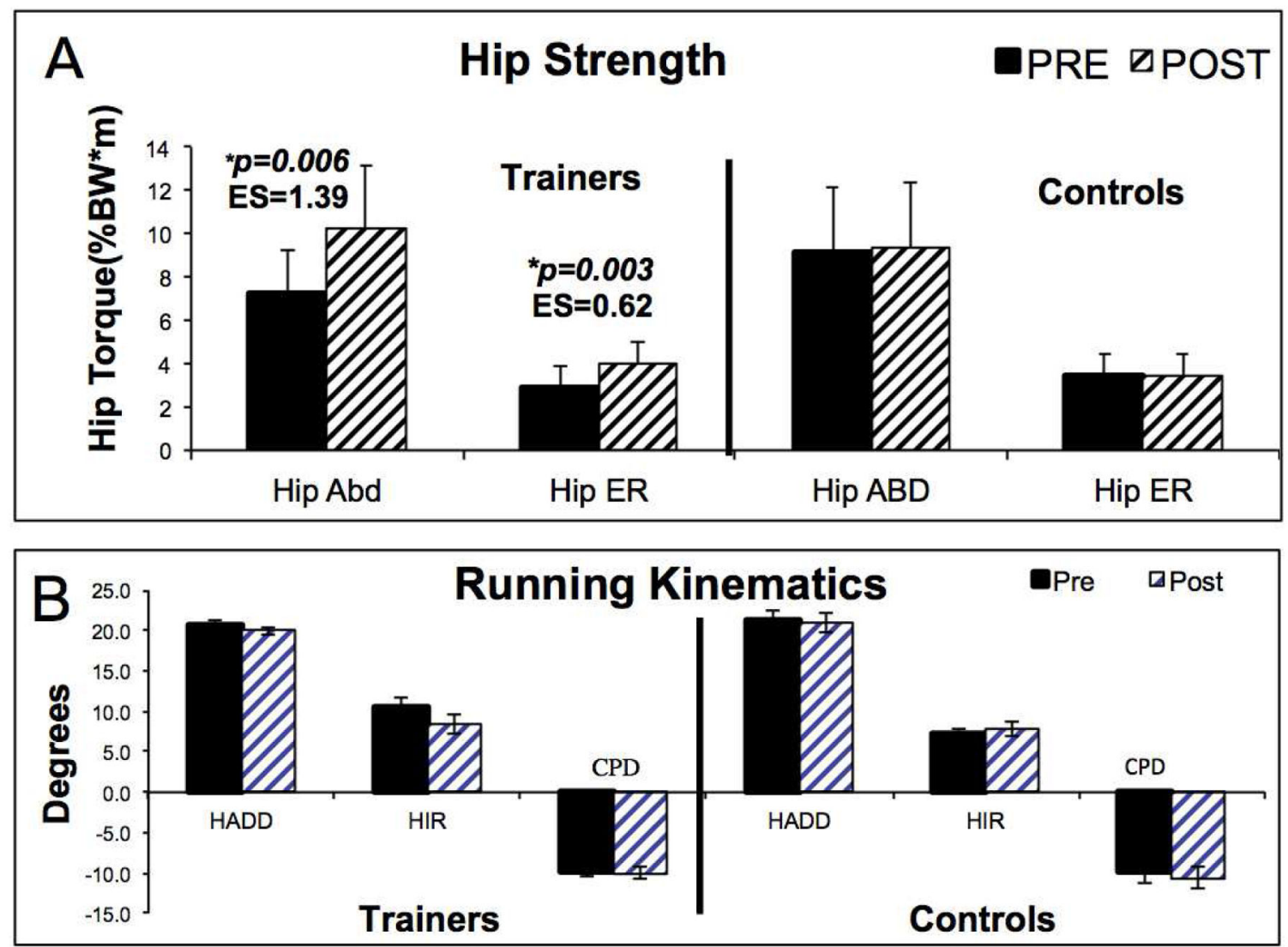

Figure 5.

Results of study by Willy and Davis. ${ }^{16}$. A. Comparison of hip strength values. Note the significant change in the Trainer group. B. Comparison of pre- and post-strengthening hip kinematics in the Trainer group. Note the lack of change in hip movement patterns, despite the increase in strength noted in this group. 


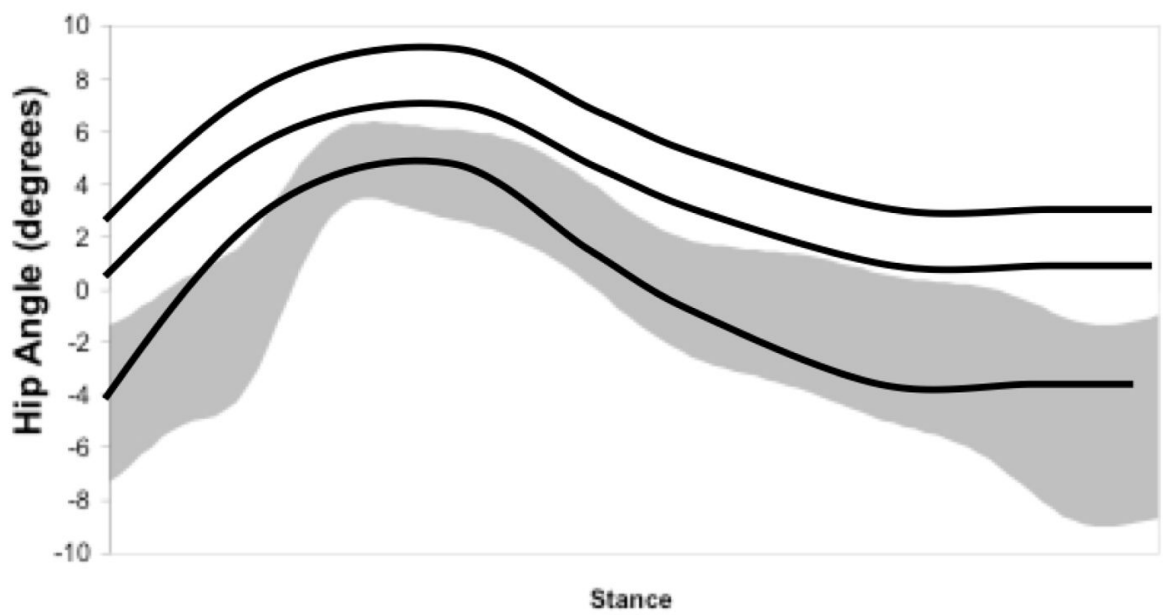

Figure 6.

Real-time feedback of hip adduction angle during a retraining session. The shaded region represents +/- 1 standard deviation from the mean value of hip adduction based on a healthy population of runners. With each stance, the hip adduction angle is provided to the runner on a monitor in front of them. They are instructed to modulate their gluteal activation until they are able to keep their hip adduction angle within the targeted shaded region. 


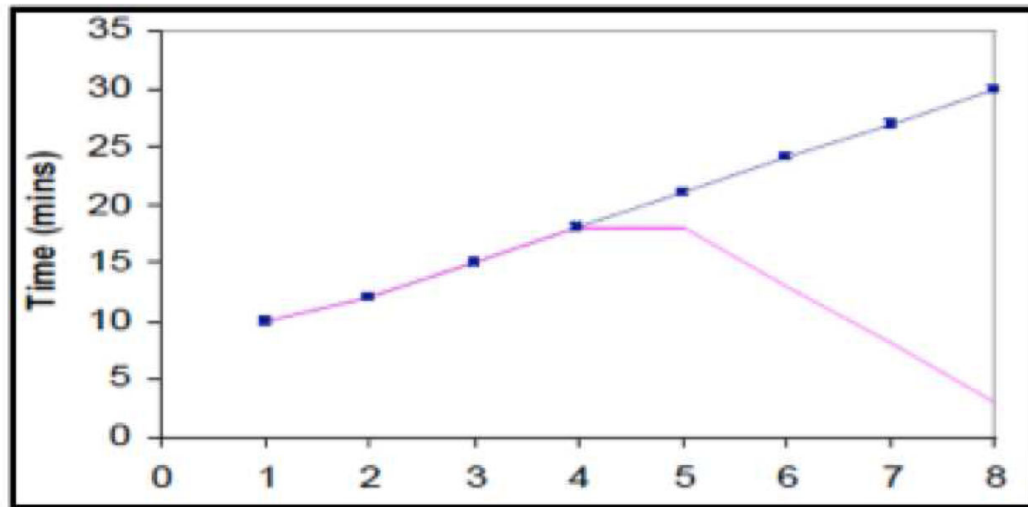

Figure 7.

Faded feedback design used in many of the gait retraining studies. Run time is increased from 10 to 30 minutes over 8 sessions. Feedback is provided $100 \%$ of the time over the first 4 sessions and then is gradually removed over the last 4 sessions. 

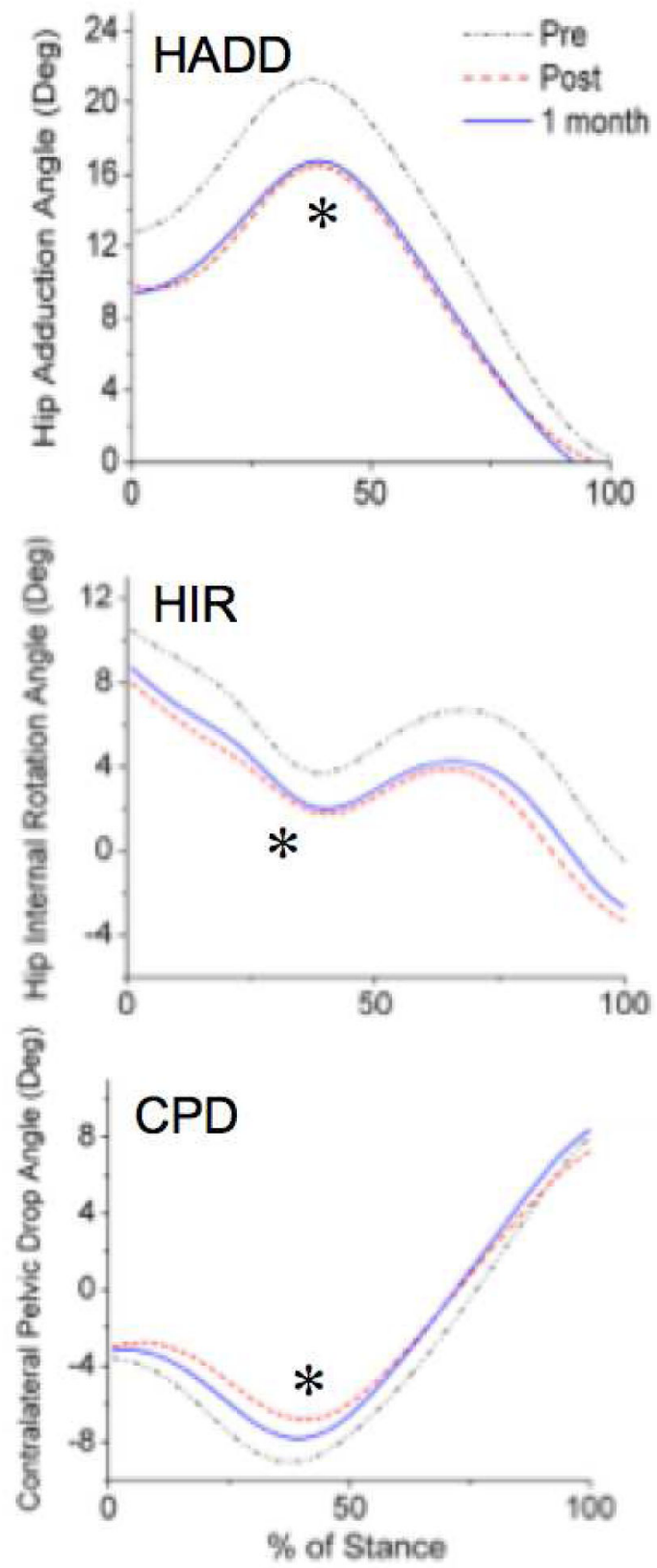

Figure 8.

Results of the Noehren et al. ${ }^{32}$ Note the reduction in hip adduction, internal rotation and contralateral pelvic drop, both at 1 and 3 month follow-ups. 


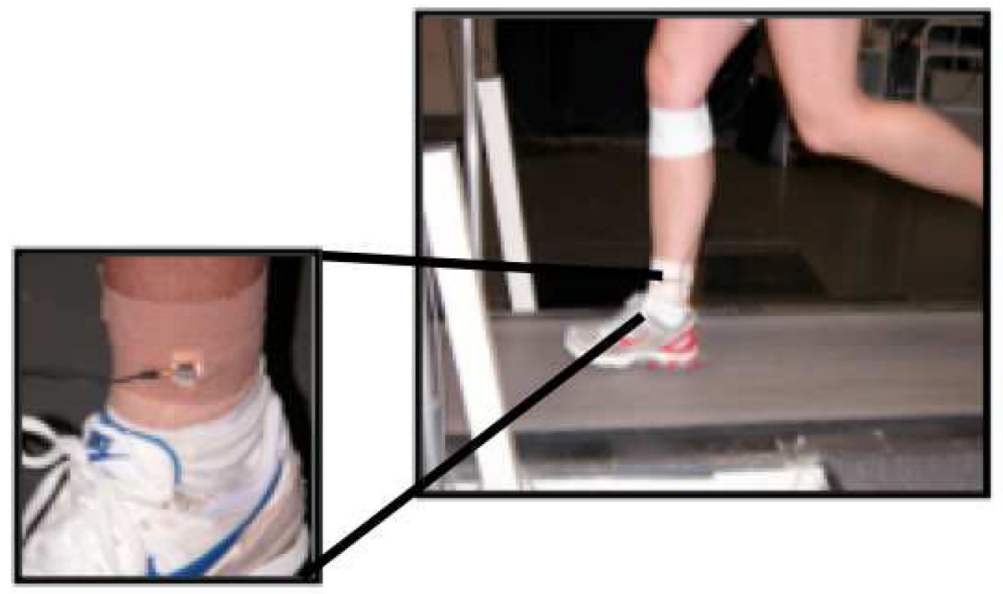

Figure 9.

Measurement of tibial shock while running. A lightweight accelerometer is tightly affixed (and overwrapped) to the distal medial tibia. 


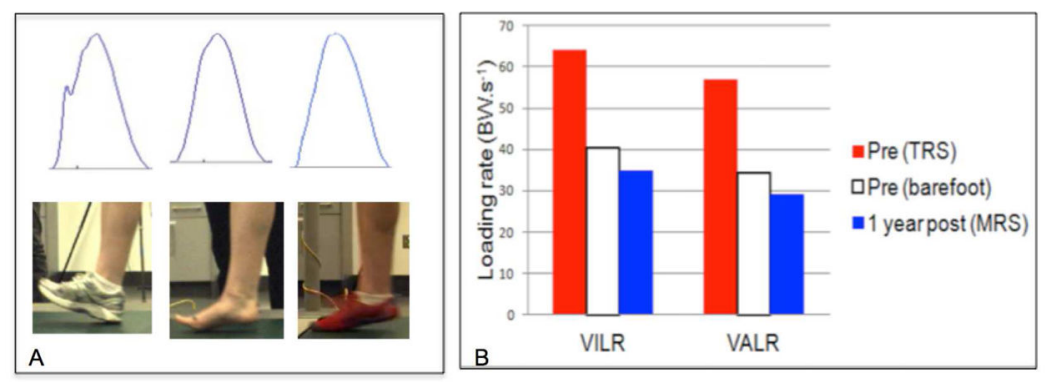

Figure 10.

Long term follow-up on a patient who underwent a supervised transition to running with a forefoot strike pattern using foot and ankle strengthening and gait retraining. A. Footstrike pattern and associated vertical ground reaction forces at left: Baseline with shoes (RFS), middle: Baseline, barefoot (FFS), and right: 1 yr followup, minimal shoes (FFS). B. Vertical instantaneous and average load rates at baseline (PRE) when patient was running with shoes (red), barefoot (white) and 1-year post when running in her minimal shoes (blue). Note reduction of her load rates when barefoot at baseline and in her minimal shoes at 1 yr post. 


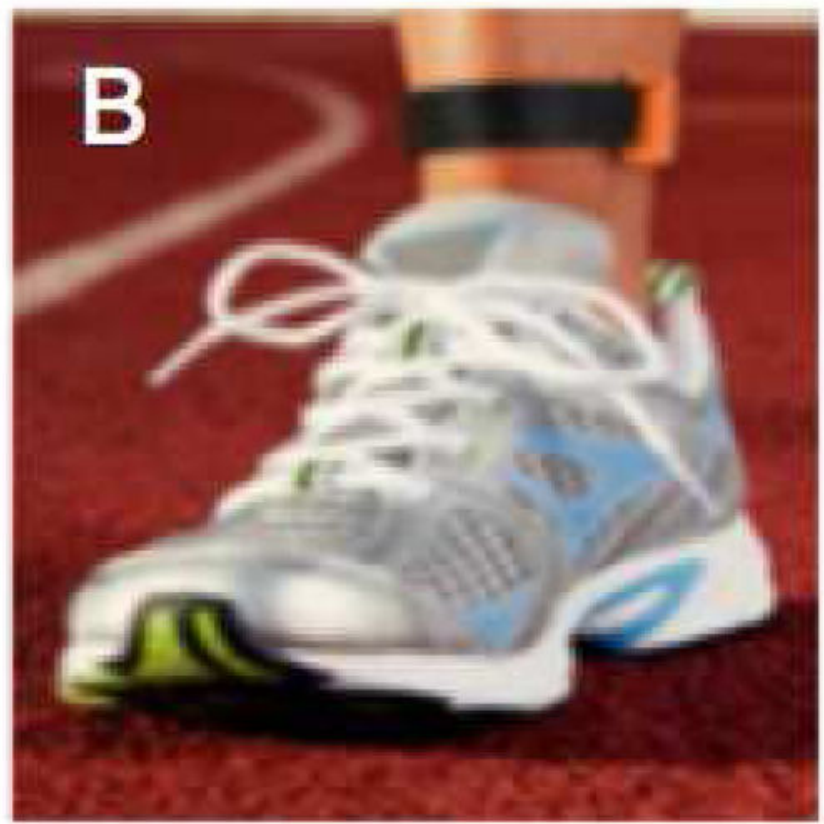

Figure 11.

A. An example of an inertial measurement unit (IMU) and B. attached to the ankle. These devices facilitate the measurement of gait in runners in their natural environment. 